\title{
Animal Research that Respects Animal Rights: Extending Requirements for Research with Humans to Animals
}

\author{
Angela K. Martin \\ Department of Philosophy, University of Basel, 4051, Basel, Switzerland \\ Corresponding author: Email. angela.martin@unibas.ch
}

\begin{abstract}
The purpose of this article is to show that animal rights are not necessarily at odds with the use of animals for research. If animals hold basic moral rights similar to those of humans, then we should consequently extend the ethical requirements guiding research with humans to research with animals. The article spells out how this can be done in practice by applying the seven requirements for ethical research with humans proposed by Ezekiel Emanuel, David Wendler, and Christine Grady to animal research. These requirements are (1) social value, (2) scientific validity, (3) independent review, (4) fair subject selection, (5) favorable riskbenefit ratio, (6) informed consent, and (7) respect for research subjects. In practice, this means that we must reform the practice of animal research to make it more similar to research with humans, rather than completely abolish the former. Indeed, if we ban animal research altogether, then we would also deprive animals of its potential benefits-which would be ethically problematic.
\end{abstract}

Keywords: animal research; animal rights; animal experimentation; speciesism; research ethics

\section{Introduction}

The ethics of animal experimentation is a controversial topic, currently receiving much attention from both animal researchers and bioethicists. Some argue that animal research is permissible if currently implemented research requirements are met and the research protocol is approved by an independent animal ethics committee (AEC; sometimes also called Institutional Animal Care and Use CommitteeIACUC). Others, especially defenders of animal rights such as Tom Regan and Alasdair Cochrane, defend the view that harmful animal research is immoral and should be abolished. However, both philosophers admit that nonharmful animal experimentation may be ethically justified. For example, Regan states that " $t]$ he rights view is not against research on animals, if this research does not harm these

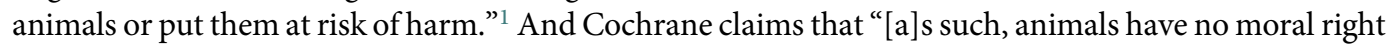
not to be used for certain purposes if their well-being is respected, and that includes their use in experimentation. If scientists experiment on animals in ways that cause no pain and that do not end in death, then such experiments are permissible." ${ }^{2}$ However, neither Regan nor Cochrane flesh out what animal research that respects animal rights would be in practice.

This article aims to close this gap in the animal rights literature by arguing that if animals have the same moral worth as humans, along with inviolable rights, then ethical research requirements and principles regulating research with humans should be extended to them as well. Indeed, research with humans-even with groups who cannot speak for themselves-is permitted, so long as some basic requirements are met. If groups unable to consent to their participation in studies were entirely excluded from research, they could not gain from its potentially beneficial discoveries. The same holds for

\footnotetext{
(C) The Author(s), 2022. Published by Cambridge University Press. This is an Open Access article, distributed under the terms of the Creative Commons Attribution licence (https://creativecommons.org/licenses/by/4.0/), which permits unrestricted re-use, distribution, and reproduction in any medium, provided the original work is properly cited.
} 
nonhuman animals: research with them should not be condemned outright, but rather conducted in a different way-viz., so that they can also potentially benefit from research results.

To be sure, there is controversy about the standards required for ethical research with humans. In order to make my argument, I rely on the list of requirements that all research with humans must meet presented by Ezekiel Emanuel, David Wendler, and Christine Grady. In their article "What Makes Clinical Research Ethical?," they list seven requirements, including (1) social value, (2) scientific validity, (3) independent review, (4) fair subject selection, (5) favorable risk-benefit ratio, (6) informed consent, and (7) respect for research subjects. ${ }^{3}$ I outline how these requirements can be applied to the case of animal research in practice; and argue that if animals have basic rights, then much higher research standards than the ones currently implemented have to be met in order for animal research to become ethically legitimate.

The thesis defended here-standards implemented for research with humans should be extended and applied to animals-is not entirely new (see, e.g., Hope Ferdoswian and Chong Choe). ${ }^{4}$ Over the last 15 years, many authors have advocated extending the principles and requirements used in research with humans to animals. However, most authors have respectively focused on a single research requirement, such as respect for autonomy, ${ }^{5,6}$ harm-benefit ratio, ${ }^{7,8}$ respect, ${ }^{9}$ or vulnerability. ${ }^{10,11}$ The aim is to present a more complete picture here.

Two recent publications present more comprehensive accounts of the research principles for animal research. In their article, “A Belmont Report for Animals," Hope Ferwodsian, Syd L. Johnson, Jane Johnson, Andrew Fenton, Adam Shriver, and John Gluck argue that the key ethical principles of the Belmont Report -that is, respect for persons and their autonomy, beneficence, justice, and special protection for vulnerable individuals and populations-should be binding for animal research. ${ }^{12}$ The authors call for an internationally binding document for animal research invoking these principles. Their project is important and laudable, and I agree with most of their conclusions. However, by focusing on the research requirements proposed in the Belmont Report, they leave aside some elements that are constitutive of ethical animal research. Their account should thus be complemented and refined with further principles.

Tom Beauchamp and David DeGrazia recently presented a more detailed account of ethical principles for animal research. ${ }^{13}$ In their book, Principles of Animal Research Ethics, they outline six moral principles that should govern animal research - three related to social benefit and three related to animal welfare:

1) the principle of no alternative method;

2) the principle of expected net benefit;

3) the principle of sufficient value to justify harm;

4) the principle of no unnecessary harm;

5) the principle of basic needs; and

6) the principle of an upper limit to harm.

Beauchamp and DeGrazia claim that these principles "can be accepted by all parties who are enthusiastic about the history and promise of animal research and all parties who are enthusiastic about vigorous protection of animal research subjects' welfare-without sacrifice of anyone's basic commitments." 14 That is, they seek a compromise between proponents of animal research and those wanting to protect animal welfare, limiting their framework to those values that most people are likely to endorse. ${ }^{15}$ Thus, their strategy is to propose principles on which animal research proponents and defenders of the animal cause can converge, with a view to forging a pragmatic compromise that will substantially improve animal research. The upshot is that they provide a rather pragmatic framework for ethical animal research, but are not concerned with the question of what ethical research would look like if animals had fundamental rights.

As a result, there is still no full and detailed account that fleshes out what animal research should look like if animals have fundamental rights. Accordingly, the aim here is to outline the principles that should govern animal research if we accept the premise that animals have basic rights. ${ }^{16}$

The argument proceeds as follows: First, I discuss the fundamental rights of animals. Since the claim of this paper is a conditional one-viz., if animals have moral rights, then the ethical requirements, 
standards, and principles governing research with humans should be applied to animal research-a clear understanding of animals' rights is needed. If animals have, for example, a right to live or a right to bodily integrity, this will influence what researchers are allowed to do with them. That is, the rights of animals are listed that should be taken into consideration when conducting research. Second, the standards guiding research with humans are examined, thereby relying on the requirements outlined by Emanuel, Wendler, and Grady. Lastly, it is shown how their seven requirements can be applied to animal research in practice, and it is outlined what this would mean for the future of animal experimentation.

\section{The Rights of Animals}

In the last 40 years, many animal ethicists have argued that not only do animals matter morally, but they also have the same inherent worth or moral status as humans. This proposition was often advanced by rejecting speciesism. The term "speciesism" was originally introduced by Richard Ryder and taken up by Peter Singer, who presented the first detailed analysis in Animal Liberation: "Speciesism — the word is not an attractive one, but I can think of no better term-is a prejudice or attitude of bias in favor of the interests of members of one's own species and against those of members of other species." ${ }^{17}$ That is, speciesist behavior entails "an unjustified disadvantageous consideration or treatment of those who are not classified as belonging to one or more particular species." ${ }^{18}$ Correspondingly, antispeciesists claim that a discrimination based merely on species membership is morally problematic, as species membership is morally irrelevant. Thus, if one aims for a non-speciesist treatment of animals, one has to consider these beings, their interests, and their rights in an unbiased way.

In what follows, I do not argue in favor of animal rights per se. For the sake of the argument, I start from the premise that animals have inviolable moral rights, similar to humans'. The aims in what follows are threefold: first, to show that if animals have rights, then a complete halt to animal research is unnecessary; second, to specify the fundamental rights of animals that are relevant to animal research; and third, to adumbrate what animal research could look like if we were to take animals' rights seriously.

A useful starting-point to determine animals' rights are the "Five Freedoms of Animal Welfare" developed by the United Kingdom Farm Animal Welfare Council (FAWC) in 1979. They include:

1) Freedom from hunger and thirst—by ready access to fresh water and a diet to maintain full health and vigor.

2) Freedom from discomfort-by providing an appropriate environment including shelter and a comfortable resting area.

3) Freedom from pain, injury, or disease-by prevention or rapid diagnosis and treatment.

4) Freedom to express normal behavior-by providing sufficient space, proper facilities and company of the animal's own kind.

5) Freedom from fear and distress-by ensuring conditions and treatment which avoid mental suffering. ${ }^{19}$

These Five Freedoms can be deemed individually necessary and jointly sufficient as a framework for the analysis of animal welfare. If all these requirements are fulfilled, this should lead to a high overall level of animal welfare. ${ }^{20}$

Furthermore, the Five Freedoms can be rephrased as rights. This presupposes that the Five Freedoms involve corresponding duties, which does seem to be the case: farmers are required to respect the Five Freedoms and thus their animals' basic needs. ${ }^{21}$ Likewise, Clare McCausland has argued that the Five Freedoms can be regarded as welfare rights. ${ }^{22}$ Steven McCulloch concludes: “Taken together, FAWC's mixed ethical approach, together with the correlative nature of rights and duties, suggests that FAWC's prescription is very close to a recommendation of moral rights for farm animals." ${ }^{23}$ Accordingly, animals can hold rights commensurate with their welfare interests covered by the Five Freedoms.

Although the Five Freedoms were originally developed for farmers and animal husbandry, they can also be applied to animal research-implying that researchers have a duty to respect the Five Freedoms. 
Research animals depend exclusively on laboratory staff members for meeting their basic needs, which results in claims for the provision of food and entertainment possibilities. That is, research animals have the right to live free from hunger and thirst, discomfort, pain, distress, and so forth, and they have the right to pursue species-typical behavior. In practice, this means that laboratory stuff and researchers must provide animals with enough space, companionship (where indicated) and possibilities for entertainment, to ensure their physical and mental well-being.

One aspect neglected by the Five Freedoms is animals' deaths. Most currently implemented animal research guidelines are concerned with minimizing animal suffering, and animals' deaths before, during, and after experiments is less frequently discussed as an ethical issue. However, according to many animal ethicists, not only animals' welfare, but also their deaths matter morally. Death forecloses future opportunities for the satisfaction of interests and is thus a harm (by deprivation) to sentient animals. That is, although most sentient animals do not have a concept of death (similarly to infants or severely cognitively disabled humans), their life has a value for them: they have an interest in continued existence, insofar as it allows them to live through future pleasant experiences. ${ }^{24,25,26,27}$ Moral agents have thus a pro tanto duty not to end animals' lives unnecessarily and prematurely. Therefore, we can add the freedom to continue living one's life to the list of the Five Freedoms.

If we phrase these freedoms in terms of rights, this leads us to the following list:

1) Right to be free from hunger and thirst;

2) Right to be free from discomfort;

3) Right to be free from pain, injury, or disease;

4) Right to be free to express normal behavior;

5) Right to be free from fear and distress;

6) Right to continued existence.

If one accepts that animals have these rights, there are consequences for their permissible use in medical research. However, as will be argued in what follows, these rights do not imply that research with animals is always ethically problematic. Many human research subjects find themselves in situations similar to those of research animals. For example, they live in extreme dependency on others (as in the case of prisoners or small children), or they are unable to understand what medical research is so as to consent to it (like severely mentally handicapped individuals or infants). Nevertheless, research with them is not categorically forbidden. Rather, if special protective measures are taken and certain rules are respected, research with them may be ethically permissible. This allows them, in turn, to profit from the potential scientific benefits of the studies in which they are involved. The same principle should apply to research with animals.

\section{Requirements for Ethical Research with Humans}

The objective of medical research is to promote society's interest in health, knowledge, and well-being at large. In order to avoid the exploitation of research participants in the pursuit of these aims, protective ethical requirements for research have been put in place. These research requirements should be sensitive to humans' basic rights, such as our right to bodily integrity and our right to respectful treatment. Furthermore, medical research should not override individuals' basic rights for the sake of the majority.

In addition, some requirements in place for studies on humans are concerned with the form of the research, rather than the basic rights of the enrolled participants. Scientific validity is an example: research that does not meet this requirement is void, as the results are useless from a scientific perspective. The same applies to independent review by Institutional Review Boards (IRBs). IRBs ensure that the study pursues an important aim, that the methodology is sound, and that research participants are chosen fairly (not due to their membership of a socially salient group, for example). Thus, research should be governed by the principles of justice and impartiality. 
After examining different research guidelines and medical research codes, Emanuel, Wendler, and Grady list seven requirements that should guide all research with humans:

1) social value;

2) scientific validity;

3) independent review;

4) fair subject selection;

5) favorable risk-benefit ratio;

6) informed consent; and

7) respect for research subjects. ${ }^{28}$

In other words, research should be socially or scientifically useful, address important societal and scientific questions, and yield valuable results for society as a whole. The results should be methodologically, statistically, and scientifically sound; that is, accepted scientific principles and methods should be used in order to produce reliable and valid results. Furthermore, the results should be described in a comprehensible way that allows for the study to be replicated. Independent review requires that the study protocol be reviewed and approved by an independent committee charged with investigating whether the study addresses a scientifically important question and is thus indispensable. It also determines whether the study's design is suitable for achieving the study's aims and whether the risk-benefit ratio is justified. Fair subject selection demands that justice prevail when choosing the group with which research is conducted. For example, vulnerable or stigmatized groups should not be targeted for high-risk research if the research could also be conducted with other groups. That is, researchers should choose the research participants based on the study's goals-not simply because of the greater convenience or availability of certain individuals who are willing to take high risks (e.g., due to terminal disease or poverty). Favorable risk-benefit ratio requires a just distribution of burdens and benefits: risks should be minimized and be in proportion to the expected benefits of the study (for the individual concerned and for society at large). Informed consent requires that research subjects be aware that they are participating in a study, that they understand the concomitant risks, and that they consent to their participation only after having been given all relevant information. If research subjects cannot consent due to the nature of the study (e.g., patients in emergency rooms or in a coma) or because they lack the relevant cognitive capacities for giving informed consent, then consent by proxy in the participants' best interest or in accordance with the presumed values of the individual is required. Furthermore, the risk should be minimized to an acceptable level in such cases. Finally, respect for research subjects means that participants are permitted to withdraw from the study at any time, that their privacy is protected by confidentiality, that they are informed of newly discovered risks, benefits and the study's results, and that their welfare is maintained throughout the study. In the following, I argue that these same requirements should be extended to research animals, and I show how this can be done in practice.

\section{Extending Research Requirements for Humans to Animals}

If we want to respect the rights of animals outlined above-e.g., to be free from discomfort, disease, fear, distress and pain, to express normal behavior, and to enjoy continued existence-this precondition restricts what we are consequently allowed to do with them in research. Inducing diseases in animals, for example, would no longer be permissible. Experiments that cause suffering or lead to the death of the animals would be ethically problematic. And so would experiments that severely restrict animals' opportunity to pursue species-typical behavior, such as maternal deprivation studies.

However, these restrictions do not rule out other forms of nonharmful animal research. Animals cannot speak up for themselves and give informed consent to their participation in medical research, as they lack the requisite cognitive capacities. For this reason, they share similarities with many groups who cannot consent to research, such as infants, persons in a coma or severely cognitively disabled 
individuals. On the other hand, these groups should not necessarily be excluded from research, since this would deprive them of all the potential benefits that the research could bring to them. ${ }^{29}$ Indeed, we already permit research that involves little or no risk and that is not harmful with groups unable to consent, so long as there is consent by proxy in their best interest. If animals have basic rights similar to those of humans, then the same principle should apply to them: nonharmful and low-risk research with them should be permissible, since a complete abolition of animal research would deprive animals of research results that are beneficial to them individually, or to their species.

Emanuel, Wendler, and Grady lay out ethical research requirements for studies with diverse populations, including vulnerable groups. If animals have rights similar to humans', then we have good reasons to extend these requirements to research animals. Here, I outline how this can be done in practice. Note that three conditions are already required for animal research in most countries, namely social and scientific value, scientific validity, and independent review. ${ }^{30}$ While necessary, these requirements are insufficient for ethical animal research. Further elements are needed that (i) guarantee animals' welfare throughout studies and (ii) provide formal requirements for any research with sentient beings, regardless of species.

\section{Social Value}

Let us turn first to social and scientific value. As in the case of research with humans, research with animals should only be conducted if it addresses important societal and scientific questions, yields valuable results for society at large, generates new knowledge, or replicates previous results. Most animal research studies start from the assumption that the expected value should only or primarily benefit humans. However, if animals have fundamental rights, this is problematic: if studies are pursued on and with animals, then the benefits should also be useful to individual animals or their species, not solely to humans. If animal research is solely responsive to humans' health priorities, then animals carry the undue burden of being used in research from which they do not benefit, which is speciesist. Yet many domesticated animals form part of our society, as humans live with them and benefit from inter-species relationships. Correspondingly, these animals' interests and health priorities should also be considered in the common good. In practice, this means that when establishing research protocols, the beneficiaries of the study's results must be identified. If they are solely a small and already over-represented group, or if the research does not address any health priority of a large group, then the study's aims and the allocation of resources to this end should be reconsidered.

A problem concerning social and scientific values arises due to the lack of obligatory registries for past and ongoing animal experiments, as is required for research with humans. Often, negative results from animal research are not published. This may lead some research groups to undertake studies that were already conducted by other colleagues, but not published. That is, some of the studies conducted on animals fail to generate new knowledge. Although some administrative and practical difficulties would have to be surmounted to establish such registries, ${ }^{31}$ they may be useful for preventing the multiplication of already-conducted experiments which did not result in any new knowledge. That is, such publicly accessible registries could reduce publication bias. ${ }^{32,33,34,35}$

\section{Scientific Validity}

Scientific validity is commonly accepted as an important requirement for animal research—not only for scientific reasons, but also for ethical ones:

[...] if poorly conducted studies produce unreliable findings, any suffering endured by animals loses its moral justification because their use cannot possibly contribute towards clinical benefit. Non-publication of animal studies is similarly unethical because the animals involved cannot contribute towards the accumulation of knowledge and because non-publication may result in further, unnecessary animal and human experiments. ${ }^{36}$ 
That is, a sufficient number of animals should be enrolled in the study to yield reliable and statistically sound results; in the published articles, experiments need to be described in a comprehensible way, enabling other researchers to replicate the study. This presupposes a detailed description of the experiments-including the sex, age, and health-status of the animals involved, along with the method and details of the statistical analysis. To avoid confirmation bias, the studies should be blinded and randomized.

The requirement of scientific validity is not always perfectly respected-in research with humans and with animals-which is why some researchers talk about a "crisis of reproducibility" in many fields, including animal research. ${ }^{37,38,39}$ If research with animals is conducted, it should be ensured by the researchers, AECs, and journal editors that the methods and statistical analysis are well described and sound. For example, this can be done by offering researchers better training about methods and potential biases, as well as by making it obligatory for all articles accepted for publication and involving animal research to follow the ARRIVE [Animals in Research: Reporting In Vivo Experiments] guidelines for reporting animal research. ${ }^{40}$

\section{Independent Review}

A further requirement for research with humans is independent review by IRBs. This is also an established requirement for animal research in most countries. AECs evaluate study protocols and decide whether the research should be approved. However, there are several issues with the evaluation processes for animal research. First, AECs are not yet obligatory in all countries. Second, AECs do not necessarily consist of independent evaluators, as required for IRBs. For example, in the United States, many AECs may be biased because they consist of animal researchers and veterinarians who themselves undertake animal research and thus depend on it for their own research ${ }^{41}$; in consequence, they may not be impartial in their evaluations. Third, different AECs often apply divergent standards when it comes to approving the very same studies. ${ }^{42}$ Fourth, AECs frequently do not follow international guidelines, but national ones; hence, approval by an AEC in one country may imply very different protection standards than in another. Finally, there is evidence that the reporting of methods in research protocols is often insufficient, but nevertheless approved by AECs due to implicit confidence rather than to evidence of scientific rigor. ${ }^{43}$

However, this does not mean that AECs are problematic per se and should be abandoned. Rather, they should be reformed. Both AECs and IRBs fulfill the important role of evaluating studies' aims and methods as well as approving the involved research populations. In order to adequately fulfill this role, AECs need clearer criteria for ethical animal research. That is, binding international guidelines for ethically acceptable research with animals should be established-as is already the case for research with humans. Such guidelines would make the evaluation process less arbitrary. Furthermore, AEC members should be completely independent of the research institution in order not to be unduly influenced or biased, and it should be ensured that at least one trained ethicist is involved in the evaluation process. ${ }^{44}$ Finally, if animals have fundamental rights, then AECs should consider these in a nonspeciesist way. This argument is further developed in the next section.

\section{Fair Subject Selection}

Currently, animals are exposed to more of research's risks than humans, while not expecting any of its benefits for themselves. As Chong Choe Smith writes: “[...] nonhuman animals bear a disproportionate share of the burdens of research without a showing of sufficient justification-for example, a showing that there are morally relevant and significant differences to justify the ethical use of nonhuman animals in research." ${ }^{25}$ This may especially concern animal species we know little about. A lack of knowledge about some species' needs or capacity to suffer (e.g., insects) may lead to their interests' being summarily discarded or simply ignored. Researchers may be tempted to use these animals more often or in high-risk research which supposedly does not afflict this group, thereby placing an unduly heavy burden on them.

The same applies to research that is inconsistently regulated, or not at all. Some species (such as rats, mice, fish, birds, or amphibians) are not always covered by animal welfare regulations in certain 
countries (e.g., the United States). However, since animals of the same species presumably do not differ regarding their experiential welfare, international guidelines should protect the interests of all animals of the same species in similar ways all around the world. That is, binding international guidelines for ethical animal research are needed-ones that require similar standards for the same species of sentient animals, regardless of where the research is being conducted. Having such internationally recognized, clear-cut criteria for ethical animal research would, in turn, also simplify the evaluation process for AECs.

In principle, researchers should always choose the best model for their research. In practice, however, some species, such as rodents, may be more frequently sought out for studies because they are easily available and do not demand a huge investment of time and money. However, if they are an inappropriate model for the condition being investigated, or if they are not afflicted by the condition being investigated, then the study should not be conducted with them.

Hence, to avoid overburdening animals in research, it must be ensured that animals are only included in research that serves them individually or their species. That is, AECs would need to keep an eye on the animal population chosen for study purposes, and demand a justification for why a specific species was singled out. In practice, this means that AECs should treat animals in research in ways similar to other society members who cannot express themselves for or against their participation in research, such as persons in a coma, infants, or severely cognitively disabled individuals. In the latter type of cases, IRBs have to check whether enrolling this group is necessary for achieving the study aims, or whether these particular groups were chosen due to mere convenience, for example. The same should apply to research animals, as their situation-dependency and inability to speak out for themselves-is similar to these populations'.

\section{Favorable Risk-Benefit Ratio}

We refrain from conducting studies with human research subjects if the harm and risk involved is too high for them-even if the results obtained would be highly beneficial to society at large. Neither do we accept the death of human research subjects as a normal consequence of a study. That is, studies with humans must have a favorable risk-benefit ratio. If animals have fundamental rights, then the same principle should apply to research with them. So far, however, animals often do not benefit from the research undertaken on them: the results obtained usually serve the human species exclusively.

Note, though, that research with humans may sometimes be permissible even if the research subjects themselves do not benefit from it, as long as the results obtained would be useful to the general population. Yet this is only the case if the research participants can consent to the study and are informed about the concomitant risks, or if the risk threshold is low so that participation is permissible without direct consent, that is, if the participants are unable to give informed consent and participation is in their interest. The acceptable risk-threshold in the case of humans unable to give informed consent remains disputed in the literature. ${ }^{46,47}$ Defining a risk-threshold for animals is not the aim here and would be a task for separate project. However, what can be noted is that there should be some upper risk threshold for animal research, as is the case of research with humans:

One way of thinking about problems of upper limits is that if it is not justified to exceed fixed upper levels of pain, suffering, and distress with nonconsenting human subjects in nontherapeutic research, and if animal subjects are relevantly similar to human subjects in the relevant respects, then exceeding the same levels of pain, suffering, and distress would likewise not be justified in the use of animals in research-or at least a justification would be required to show why what is unjustified with human subjects is justified with animal subjects. When human interests and animal interests are relevantly similar and their welfare is contingent on not being constrained, coerced, deprived of basic needs, and placed in pain or terror, it is difficult to see what, if anything, would justify treating the interests of animals as dissimilar to human interests. ${ }^{48}$ 
Although to date only few guidelines, such as the Preamble 23 of Directive 2010/63/EU prescribe an upper limit and a favorable risk-benefit ratio for animals, ${ }^{49}$ it should become a requirement in all animal research guidelines.

\section{Informed Consent}

The extension of informed consent to research animals is quite frequently discussed in the literature. Hope Ferdowsian and Chong Choe, for example, state: "[...] although many animals exhibit intelligence, rationality, and maturity, language barriers prohibit informed consent." ${ }^{\circ 0}$ Holly Kantin and David Wendler talk of "the lack of a common language," ${ }^{11}$ and Jane Johnson and Neal Barnard mention "communication barriers" 52 insofar as animals cannot tell us what kind of research environment is appropriate for them. However, these characterizations are misleading, insofar as the requirement of informed consent can never apply to animals: most animals could never possibly fulfill its ascriptionconditions. The concept of language barriers suggests that if animals could talk, then the problem would be resolved. But much more than merely language and communication barriers are at stake when giving informed consent-namely, cognitive capacities such as rationality, the ability to know how to act intentionally in one's best interest in the long term, understanding complex circumstances, and the like. As Richard Healey and Angie Pepper put it: animals are incapable of giving informed consent because they "cannot understand, form, and communicate complex intentions about normative concepts like rights and duties." 53 That is, animals cannot waive rights regarding themselves and their bodies and thus authorize others to undertake an otherwise impermissible action (such as administering a drug). Animals are thus incapable of giving informed consent to their participation in medical research.

Nonetheless, there is another understanding of informed consent that could be applied to the case of animal research, namely assent and dissent. Animals have various preferences, which they can express. For example, animals can show-with the help of humans and a trial-and-error system-their food preferences, or, in the case of dogs, which walking route they wish to take with their human guardian. I argued earlier that animals have a basic right to express normal behavior. Part of animals' normal behavior is their ability to freely pursue their own interests and preferences. To act upon their will-to move freely, to curiously discover a new area or to freely choose with whom they interact-constitutes a significant part of a good life for many animals. That is, many animals can express their will through their actions and in interactions with other beings-and this capacity should be respected. Applied to the case of animal research, this means that animals can show whether they wish to partake in research or not. Thus, there is a way to reformulate the idea of informed consent in research animals:

The fact that investigators are required to solicit the assent and respect the dissent of human subjects who are unable to provide informed consent suggests that animals' inability to provide informed consent does not provide a justification for failing to take into account their preferences regarding whether they participate in research. ${ }^{54}$

Kantin and Wendler distinguish welfare- and agency-based reasons for why researchers should respect animals' preferences to withdraw from or participate in research. Preferences can mirror individuals' welfare, which provides a pro tanto reason to take them into account. Hence, researchers should observe if animals show dissent (e.g., in the form of discomfort or pain), with the aim of maintaining their welfare. ${ }^{55}$ Although it may be difficult to determine the exact source of unease in research animals (since it may also be due to fear, hunger, and the like), paying attention to dissenting behavioral cues can help to determine whether the harm experienced by the research animals exceeds an allowable upper limit, and corresponding measures could be taken to minimize suffering. That is, respect for sustained dissent can minimize the overall harm incurred by research animals.

Agency-based reasons are concerned with respect for individuals' capacity to freely choose what they prefer, regardless of whether it is beneficial or detrimental to their well-being. Agency has intrinsic value insofar as it matters for forming and shaping one's life in the way one wishes. This capacity for agency can 
sometimes be found in some animals (e.g., great apes, dolphins, and possibly elephants). Kantin and Wendler infer from this fact that, "[...] at a minimum, it seems plausible that investigators have an obligation to make sure that no animal to whom agency-based reasons apply has her dissent disrespected on a consistent basis." ${ }^{56}$ In practice, this means that different methods or analgesia should perhaps be deployed, or that strongly dissenting animals should be excluded from research.

In some cases, it is also possible to extend the principle of assent to research animals, such as when animals can freely choose to join the laboratory of their own will and when it pleases them, for example in the case of behavioral studies. Under animals' assent, I understand here animals' approval of what is happening to them. This can manifest itself in the form of animals' not showing disapproval or resistance, their showing approval to what is happening to them, or their affirmative behavior, such as when they join a study setting deliberately. ${ }^{57}$ An example are chimpanzees in reserves or sanctuaries who participate in studies involving video games which test their cognitive capacities; they engage in this research of their own will, since it presents them with a welcome distraction from their daily life.

In summary, this means that close attention should be paid to the behavior and preferences of individual animals during studies. Researchers should test whether animals show assent to their study participation, and steps should be taken if dissent is perceived. In practice, this implies that many painful experiments conducted on animals would no longer be possible.

\section{Respect for Research Subjects}

As their last requirement, Emanuel, Wendler, and Grady list "respect for research subjects." The first aspect of this criterion involves permitting withdrawal from the research anytime, a point already covered in the previous section on animals' assent and dissent. Furthermore, Emanuel, Wendler, and Grady mention the need to protect privacy through confidentiality, to provide information about newly discovered risks or benefits, and to inform research subjects of the clinical research's results. These requirements do not seem relevant to the case of animals. However, there is a fifth requirement that does matter, namely, that researchers maintain the welfare of their research subjects. This can be described as beneficence. Current research practices often fall short of this requirement. For example, it is a common practice to induce harmful conditions and diseases in animals (such as cancer or lameness) - a practice we should never accept in the case of humans. If animals have a right to remain free of disease and to benefit from bodily integrity, then the same protection should apply to their case: causing them deliberately harmful conditions (such as, e.g., cancer) would be morally impermissible.

This leads us to consider another problematic practice, namely bringing animals into existence merely to serve research purposes. Many animals are bred for research, and they will normally spend their whole lives in research settings, afflicted by disease or other painful conditions while being used in harmful experiments until they die. These animals are not brought into existence for their own sake; rather, they are exclusively bred as means to the end of the research, and usually they will experience a rather low quality of life. If animals have basic rights, such pure instrumentalization is morally problematic. We usually think that it is morally reprehensible to bring children into existence solely as mere means to an end, such as serving their parents. The same should hold for research animals, if they have basic moral rights. As Sue Donaldson and Will Kymlicka note regarding companion animals: "Humans may bring dogs into their lives for pleasure (and company, love, and inspiration), but this is compatible with dogs existing in and for themselves) as it is in the case of humans. ${ }^{\text {" } 8}$ In practice, humans can still bring domesticated animals into existence, but these animals always have to be valued for themselves: they cannot exclusively be bred for the purpose of animal research alone, being deliberately infected with diseases or having other harmful conditions inflicted upon them. This means that nonharmful or minimally invasive research with already-existing sick animals may be ethically permissible, as long as it is in the interest of these animals to participate in a given study.

Lastly, respect for animals also means that their right to life must be respected: laboratory animals should not automatically be put to death once the study ends, unless unavoidable suffering or pain due to natural causes makes euthanasia necessary. Rather, steps should be taken to allow these animals a fulfilled life in sanctuaries or homes. That is, animals should only be put to death if it is in their own best 
interest and unavoidable for reducing suffering that cannot be alleviated otherwise. ${ }^{59}$ A possible option is to rehome animals once a study is finished, that is, to find caretakers or sanctuaries for animals used in research ${ }^{60}$ - a practice already suggested for some species by the European Directive on the protection of animals used for scientific purposes. ${ }^{61}$

\section{Conclusion}

In this article, I have defended the view that if animals have moral rights, then animal research should not necessarily be abolished, but should rather be completely reformed by modeling it on ethical research with humans. If we entirely excluded animals from research, then we would deprive them of potential benefits to them or their species. Taking the Five Freedoms as a basis, I fleshed out animals' basic rights and argued that requirements already in place for ethical research with humans should be extended to animal research. If research with humans who cannot speak up for themselves and consent to their participation in studies may be conducted as long as some requirements are met and the risk-level is low, I contended, then the same conditions should apply to animals' involvement in research. Moreover, I outlined how this can best be done in practice, by assessing how the requirements of social and scientific value, scientific validity, independent review, fair subject-selection, harm-benefit ratio, informed consent, and respect could be applied to animal research.

Given all these considerations, it appears that only noninvasive animal research, some behavioral studies where animals are merely observed, practices involving little or no harm and stress, and the use of the animals after their natural death are ethically permissible. Further cases of admissible research include: "the disease or condition being investigated is one that naturally occurs in the study animal; the animal enrolled in the experiment is already afflicted with that disease or condition; and participation in the research offers the chance of benefit (or no more than minimal risk) to the individual participant." 62

Note that the list of rights and research requirements presented here is a rather basic one. That is, the rights and requirements presented form a minimal threshold for ethically acceptable animal research. Other, complementary principles may be added to this list, such as protections for particularly vulnerable groups of animals (e.g., animal species who need special provisions when in research settings due to their specific needs, which may be harder to meet or are more likely to be overlooked). However, the rights and requirements presented here are meant to establish the most basic conditions animal research should meet on the supposition that animals have rights.

To be sure, there may be disagreement about some of the animal rights and requirements I defend in this article. Some people may argue, for example, that the right to continued existence is too demanding, and that animals lack such a right. However, even if I deem the list of requirements presented here jointly necessary for ethical animal research, there remain possible applications of research requirements that are compatible with animals' deaths. That is, given that we live in a predominantly speciesist world, even the extension of just one of these requirements would already be a step in the right direction. Hence even if one did not accept one or several of the rights or requirements outlined here, then there would nonetheless be other lessons to be learned from the application of the remaining research requirements and rights to the case of animal research.

Acknowledgments. The author wishes to thank the participants of the annual conference of the Chair of Theoretical Philosophy at the University of Basel, as well as the participants of the colloquium in practical philosophy at the University of Berne for their helpful comments on previous versions of this article. This work was supported by the Swiss National Science Foundation (Grant number 179826).

\section{Notes}

1. Regan T. The Case for Animal Rights. Berkeley: University of California Press; 2004.

2. Cochrane A. Animal Rights Without Liberation: Applied Ethics and Human Obligations. New York: Columbia University Press; 2012. 
3. Emanuel EJ, Wendler D, Grady C. What makes clinical research ethical? JAMA 2000;283 (20):2701-11.

4. Ferdowsian $\mathrm{H}$, Choe $\mathrm{C}$. Extending human research protections to non-human animals. In: Corbey $\mathrm{R}$, Lanjouw A, eds. The Politics of Species. Reshaping our Relationships with Other Animals. Cambridge: Cambridge University Press; 2013, at 232-40.

5. Kantin H, Wendler D. Is there a role for assent or dissent in animal research? Cambridge Quarterly of Healthcare Ethics 2015;24(4):459-72.

6. Fenton A. Can a chimp say "no"? Reenvisioning chimpanzee dissent in harmful research. Cambridge Quarterly of Healthcare Ethics 2014;23(2):130-9.

7. Arnason G, Clausen J. On balance: weighing harms and benefits in fundamental neurological research using nonhuman primates. Medicine, Health Care, and Philosophy 2016;19(2):229-37.

8. Ferdowsian H, Fuentes A. Harms and deprivation of benefits for nonhuman primates in research. Theoretical Medicine and Bioethics 2014;35(2):143-56.

9. Walker RL. Human and animal subjects of research: The moral significance of respect versus welfare. Theoretical Medicine and Bioethics 2006;27(4):305-31.

10. Johnson J. Vulnerable subjects? The case of nonhuman animals in experimentation. Journal of Bioethical Inquiry 2013;10:497-504.

11. Johnson J, Barnard ND. Chimpanzees as vulnerable subjects in research. Theoretical Medicine and Bioethics 2014;35:133-41.

12. Ferdowsian H, Johnson LSM, Johnson J, Fenton A, Shriver A, Gluck J. A Belmont Report for animals? Cambridge Quarterly of Healthcare Ethics 2020;29(1):19-37.

13. Beauchamp TL, DeGrazia D. Principles of Animal Research Ethics. New York: Oxford University Press; 2020.

14. DeGrazia D, Beauchamp TL. Beyond the 3 Rs to a more comprehensive framework of principles for animal research ethics. ILAR Journal 2019;59(2):ilz011.

15. Similarly, David DeGrazia and Jeff Sebo outline three principles that they deem acceptable for animal research proponents who are sympathetic to the view that animals matter morally, but who think that humans matter more than animals: an expectation of sufficient net benefit; a worthwhile-life condition; and a no-unnecessary-harm/qualified-basic-needs condition (DeGrazia D, Sebo J. Necessary conditions for morally responsible animal research. Cambridge Quarterly of Healthcare Ethics 2015;24(4):420-30).

16. A note about the types of research I am concerned with: my article deals with all types of animal experimentation in controlled settings such as laboratories. Admittedly, biological or ecological field research involving wild animals may be harmful and thus raise ethical issues (Zemanova MA. More training in animal ethics needed for European biologists. BioScience 2017;67(3):301-5); nonetheless, for present purposes, I am not concerned with the principles that should guide this type of research.

17. Singer P. Animal Liberation: The Definitive Classic of the Animal Movement. New York: Ecco Book/ Harper Perennial; 2009.

18. Horta O. What is speciesism? Journal of Agricultural and Environmental Ethics 2010;23(3):243-66.

19. Farm Animal Welfare Council (FAWC): Five Freedoms; available at https://webarchive. nationalarchives.gov.uk/20121010012427; http://www.fawc.org.uk/freedoms.htm (last accessed 30 May 2021). Another option to establish a list of animal rights would be to start from the basic rights of humans to see which ones could apply to animals. Assuming that the resulting list would probably be similar to the one I am presenting, I do not pursue this option further.

20. McCulloch SP. A critique of FAWC's Five Freedoms as a framework for the analysis of animal welfare. Journal of Agricultural and Environmental Ethics 2013;26(5):959-75.

21. FAWC. Farm Animal Welfare in Great Britain: Past, Present and Future; 2009; available at https:// assets.publishing.service.gov.uk/government/uploads/system/uploads/attachment_data/file/ 319292/Farm_Animal_Welfare_in_Great_Britain_-_Past_Present_and_Future.pdf.

22. McCausland C. The Five Freedoms of animal welfare are rights. Journal of Agricultural and Environmental Ethics 2014;27(4):649-62. 
23. McCulloch SP. On the virtue of solidarity: animal rights, animal welfarism and animals' rights to wellbeing. Journal of Animal Welfare Law 2012(Spring/Summer):5-15.

24. Sapontzis SF. Morals, Reason, and Animals. Philadelphia: Temple University Press; 1987.

25. Rollin BE. Animal Rights and Human Morality. 3rd ed. Amherst, NY: Prometheus Books; 2006.

26. DeGrazia D. Taking Animals Seriously: Mental Life and Moral Status. Cambridge: Cambridge University Press; 1996.

27. See note 1, Regan 2004.

28. See note 3, Emanuel et al. 2000.

29. Shepherd V. Research involving adults lacking capacity to consent: the impact of research regulation on 'evidence biased' medicine. BMC Medical Ethics 2016;17(1):55.

30. This is required by the United States Code Animal Welfare Act 2015; available at https://www. govinfo.gov/content/pkg/USCODE-2015-title7/html/USCODE-2015-title7-chap54.htm (last accessed 30 May 2021); Canadian Council on Animal Care research code Guide to the Care and Use of Experimental Animals, Vol. 1; 2020; available at https://www.ccac.ca/Documents/Standards/ Guidelines/Experimental_Animals_Vol1.pdf (last accessed 30 May 2021); The Directive 2010/63/ EU of the European Parliament and of the Council of 22 September 2010 on the protection of animals used for scientific purposes 2010; available at https:/eur-lex.europa.eu/eli/dir/2010/63/oj. (last accessed 30 May 2021). One could argue that weighing harms against benefits should also figure on this list of basic research standards, but since it is not an explicit requirement in the Animal Welfare Act, it is not listed here.

31. Baker D, Lidster K, Sottomayor A, Amor S, Eisen JA. Two years later: Journals are not yet enforcing the ARRIVE guidelines on reporting standards for pre-clinical animal studies. PLoS Biology 2014;12(1):e1001756.

32. Muhlhausler BS, Bloomfield FH, Gillman MW. Whole animal experiments should be more like human randomized controlled trials. PLoS Biology 2013;11(2):e1001481.

33. ter Riet G, Korevaar DA, Leenaars M, Sterk PJ, Van Noorden CJF, Bouter LM, et al. Publication bias in laboratory animal research: A survey on magnitude, drivers, consequences and potential solutions. PLoS ONE 2012;7(9):e43404.

34. Kimmelman J, Anderson JA. Should preclinical studies be registered? Nature Biotechnology 2012;30 (6):488-9.

35. Jansen of Lorkeers SJ, Doevendans PA, Chamuleau SAJ. All preclinical trials should be registered in advance in an online registry. European Journal of Clinical Investigation 2014;44(9):891-2.

36. Pound P, Bracken MB. Is animal research sufficiently evidence based to be a cornerstone of biomedical research? British Medical Journal 2014;348:g3387.

37. Begley CG, Ioannidis JPA. Reproducibility in science: Improving the standard for basic and preclinical research. Circulation Research 2015;116(1):116-26.

38. Jarvis MF, Williams M. Irreproducibility in preclinical biomedical research: Perceptions, uncertainties, and knowledge gaps. Trends in Pharmacological Sciences 2016;37(4):290-302.

39. Begley CG, Ellis LM. Drug development: Raise standards for preclinical cancer research. Nature 2012;483(7391):531-3.

40. Kilkenny C, Browne WJ, Cuthill IC, Emerson M, Altman DG. Improving bioscience research reporting: The ARRIVE guidelines for reporting animal research. PLoS Biology 2010;8(6):e1000412.

41. Hansen LA. Institution animal care and use committees need greater ethical diversity. Journal of Medical Ethics 2013;39(3):188-90.

42. Plous S, Herzog H. Animal research: Reliability of protocol reviews for animal research. Science 2001;293(5530):608-9.

43. Vogt L, Reichlin TS, Nathues C, Würbel H. Authorization of animal experiments is based on confidence rather than evidence of scientific rigor. PLoS Biology 2016;14(12):e2000598.

44. Nobis N. Why IACUCs need ethicists. ILAR Journal 2020; ilaa021.

45. Choe Smith CU. Confronting ethical permissibility in animal research: Rejecting a common assumption and extending a principle of justice. Theoretical Medicine and Bioethics 2014;35:175-85. 
46. Kopelman LM. Minimal risk as an international ethical standard in research. The Journal of Medicine and Philosophy 2004;29(3):351-78.

47. Binik A. On the minimal risk threshold in research with children. The American Journal of Bioethics 2014;14(9):3-12.

48. Beauchamp TL, Morton DB. The upper limits of pain and suffering in animal research. Cambridge Quarterly of Healthcare Ethics 2015;24(4):431-47.

49. See note 30, Directive 2010/63/EU.

50. See note 4, Ferdowsian, Choe 2013, at 232-40.

51. See note 5, Kantin, Wendler 2015, at 459-72.

52. See note 11, Johnson, Barnard 2014, at 133-41.

53. Healey R, Pepper A. Interspecies justice: Agency, self-determination, and assent. Philosophical Studies 2020;178:1223-43.

54. See note 5, Kantin, Wendler 2015, at 459-72.

55. Andrew Fenton made a similar proposition regarding dissent in research animals in general (Fenton A. Holding animal-based research to our highest ethical standards: Re-seeing two emergent laboratory practices and the ethical significance of research animal dissent. ILAR Journal 2020, ilaa014), and chimpanzees in particular (see note 6, Fenton 2014, at 130-9).

56. See note 5, Kantin, Wendler 2015, at 459-72.

57. For a detailed account of animal's assent and the conditions it needs to fulfill, see note 53, Healey, Pepper 2020. Gardar Arnason recently argued that animals are incapable of assenting because assent presupposes understanding information about what is going to happen during an event or procedure (Arnason G. The emergence and development of animal research ethics: A review with a focus on nonhuman primates. Science and Engineering Ethics 2020;26(4):2277-93). However, according to his view, animals can show acquiescence to what is happening to them. Note, although, that Arnason refers to a more demanding account of assent than Healey and Pepper, whose conception I am following here in broad terms.

58. Donaldson S, Kymlicka W. Zoopolis: A Political Theory of Animal Rights. Oxford: Oxford University Press; 2011.

59. Similarly to humans who should have access to assisted dying if they deem their suffering as too unbearable.

60. See note 55, Fenton 2020.

61. See note 30, Directive 2010/63/EU.

62. See note 11, Johnson, Barnard 2014, at 133-41.

Cite this article: Martin, A. K. 2022. Animal Research that Respects Animal Rights: Extending Requirements for Research with Humans to Animals. Cambridge Quarterly of Healthcare Ethics 31: 59-72, doi:10.1017/S0963180121000499 